Revue Française de Civilisation Britannique

\title{
La BBC et l'information sur le conflit en Irlande du Nord (1960-1995)
}

BBC and News from Northern Ireland (1960-1995)

\section{Elodie Gallet}

\section{OpenEdition}

\section{Journals}

Édition électronique

URL : https://journals.openedition.org/rfcb/7491

DOI : $10.4000 /$ rfcb.7491

ISSN : 2429-4373

Éditeur

CRECIB - Centre de recherche et d'études en civilisation britannique

Référence électronique

Elodie Gallet, « La BBC et l'information sur le conflit en Irlande du Nord (1960-1995) », Revue Française de Civilisation Britannique [En ligne], XXVI-1 | 2021, mis en ligne le 05 décembre 2020, consulté le 06 janvier 2022. URL : http://journals.openedition.org/rfcb/7491 ; DOI : https://doi.org/10.4000/rfcb.7491

Ce document a été généré automatiquement le 6 janvier 2022.

\section{c) (1)}

Revue française de civilisation britannique est mis à disposition selon les termes de la licence Creative Commons Attribution - Pas d'Utilisation Commerciale - Pas de Modification 4.0 International. 


\section{La BBC et l'information sur le conflit en Irlande du Nord (1960-1995)}

BBC and News from Northern Ireland (1960-1995)

Elodie Gallet

\section{Introduction}

1 Les conflits sont médiatiques ${ }^{1}$. Chaque événement violent donne lieu à un déchaînement des médias où se succèdent les images sensationnelles et les interviews au plus près de l'événement. La télévision britannique connaît cette tendance à l'hypermédiatisation des situations de violence, en particulier des actions terroristes ${ }^{2}$. Paradoxalement, l'approche de la BBC et des médias britanniques en général au sujet du conflit en Irlande du Nord a été différente. De nombreuses actions violentes et la mort de centaines de personnes sont passées inaperçues.

2 Le conflit en Irlande du Nord est un conflit interne, se déroulant sur le territoire national et impliquant essentiellement des civils, des groupes armés n'appartenant pas aux forces nationales, et l'armée. La télévision nationale, et donc la $\mathrm{BBC}$, pouvait-elle rester impartiale ${ }^{3}$ face à cette situation ?

3 Certes, le caractère impartial de la $\mathrm{BBC}$ en fait la réputation à l'international. Toutefois, le devoir d'impartialité, acté dans la Charte Royale définissant le fonctionnement de la $B B C$, semble difficilement conciliable avec le fait que son financement émane entièrement d'une redevance publique et donc étatique.

4 L'intensification du mouvement pour les droits civiques à la fin des années 1960 et la violence qui l'accompagna, firent connaître la situation de l'Irlande du Nord à l'ensemble du Royaume-Uni et plus largement à l'international ${ }^{4}$. L'Irlande du Nord, qui apparaissait rarement sur les ondes et les écrans depuis la Partition de l'Irlande au début des années 1920, était subitement présentée comme un terrain de violence.

Les gouvernements successifs étant conscients du pouvoir de la télévision auprès de l'opinion publique, une véritable guerre de propagande s'ensuivit puisque tous les 
acteurs impliqués dans le conflit espéraient donner leur version de l'histoire aux publics nationaux et internationaux pour justifier leur rôle dans un conflit toujours plus violent. Au niveau de la $\mathrm{BBC}$, la question récurrente fut de savoir ce qui pouvait ou non être diffusé sur le réseau régional et sur le réseau national. Elle fut attaquée, menacée, mais se défend de s'être attelée à préserver son indépendance et son intégrité journalistique. D'aucuns affirment que BBC Northern Ireland s'est imposée comme une voix critique au point de faire partie intégrante du conflit. Critique à l'égard du gouvernement nord-irlandais, en contribuant à sa chute, mais aussi critique à l'égard de la politique britannique en Irlande du Nord ${ }^{5}$.

6 Le présent article vise à tester cette assertion et à explorer, plus en général, la couverture qui a été faite par la BBC du conflit en Irlande du Nord.

7 Dans un premier temps, nous proposons de dresser un panorama depuis la recrudescence des violences à la fin des années 1960, jusqu'en 1995, fin de la période traitée marquée par les prémices du processus de paix.

8 Dans un second temps, nous nous concentrerons sur la période allant de 1988 à 1995 puisqu'elle correspond à la mise en place, par le gouvernement britannique, d'une censure audiovisuelle à l'adresse des membres et sympathisants des organisations républicaines et loyalistes répertoriées par le Prevention of Terrorism Act comme terroristes (et de trois partis politiques).

9 Enfin, nous examinerons qualitativement et quantitativement la programmation assurée sur BBC 1 et BBC 2, en nous appuyant principalement sur les documentaires et films/téléfilms diffusés sur ces chaînes entre 1988 et 1995.

\section{La représentation du conflit en Irlande du Nord à la télévision britannique avant 1988}

La médiatisation du conflit en Irlande du Nord à la BBC a connu plusieurs phases, influencées par la politique de communication du gouvernement britannique sur le sujet. Lors de de l'avènement des violences qui, en août 1969, marquèrent le début du conflit armé, dont la fin officielle remonte au 10 avril 1998 avec la signature de l'Accord de Belfast, BBC Northern Ireland, fut confrontée au choix de couvrir cette situation comme elle l'aurait fait dans un pays étranger, ou bien de procéder à une couverture partielle voire partiale des événements. Ce choix fut ultérieurement défendu par Waldo Maguire, BBC Controller for Northern Ireland, qui déclara «In the present atmosphere of hatred and fear, we have to recognise that the broadcasting of violently opposed views, passionately and offensively expressed, could have direct and immediate consequences on the streets of Belfast and Londonderry ${ }^{6}$. Plus tard, Martin Bell, correspondant de la BBC en Irlande du Nord pendant plusieurs années, déclarera :

We made a mistake (...) in 1969, in August of that year when Catholics were burned out of their homes in the Falls by Protestants who attacked them from the Shankill. The $\mathrm{BBC}$ reports then gave no indication of who these refugees were. They just spoke of refugees. The public was not to know whether they were Catholic or Protestant or who was attacking whom ${ }^{7}$.

11 La médiatisation du conflit en Irlande du Nord commença ainsi réellement à la fin des années $1960^{8}$ alors que, selon Rex Cathcart, historien de la BBC en Irlande du Nord, BBC NI avait pendant longtemps cherché à montrer une société sans divisions, au point d'exclure toute mention d'une "partition " ${ }^{9}$. Selon David Butler, avant 1968 cette 
couverture était en réalité minimale et accessoire; les journalistes ne cherchaient notamment pas à expliquer ou à enquêter sur les liens entre les différents événements, sur leurs causes profondes ${ }^{10}$. Liz Curtis avance même que jusqu'en 1968-1969 les chaînes de télévision auraient conspiré avec Stormont et Westminster pour tenir les événements en Irlande du Nord en dehors des écrans britanniques ${ }^{11}$.

Dès le déploiement des troupes en 1969 jusqu'aux cessez-le-feu de l'IRA et de groupes paramilitaires loyalistes en 1994, le gouvernement britannique adhéra publiquement à la position selon laquelle ce conflit était causé par des terroristes, en particulier les républicains, dont les motivations étaient criminelles et non politiques ${ }^{12}$. Le seul moyen de parvenir à la paix était donc de vaincre l'IRA. Diverses stratégies furent utilisées pour mener à bien cette politique de communication, lesquelles consistèrent à gérer les informations officielles relatives au conflit, à légiférer et à s'immiscer indirectement puis directement dans le travail des chaînes en télévision pour les pousser à l'autocensure ${ }^{13}$.

Dès le début des années 1970, le gouvernement britannique chercha à faire pression sur l'audiovisuel au sujet de l'Irlande du Nord. Tandis qu'une vague de violence, marquée par divers attentats perpétrés par des organisations paramilitaires, s'abattait sur le pays, plusieurs émissions diffusées à la télévision attisèrent l'hostilité du gouvernement envers les médias ${ }^{14}$. Le ministre britannique des Communications, Christopher Chataway, déclara que les acteurs de l'audiovisuel « were not required to strike a balance as between the IRA and the Ulster government or between the army and the terrorists $»^{15}$. Des députés conservateurs réclamèrent que le ministre de l'Intérieur, Reginald Maudling, impose une censure patriotique aux médias ${ }^{16}$. Ils avançaient que la $\mathrm{BBC}$ devait faire partie de la machine de propagande et que la campagne contre les paramilitaires républicains devrait l'emporter sur toute autre considération ${ }^{17}$. Ce à quoi Lord Hill, qui était alors à la tête de la $\mathrm{BBC}$, répondit :

The $\mathrm{BBC}$ and its staff abhor the terrorism of the IRA and report their campaign of murder with revulsion... as between the government and the opposition, as between the two communities in Northern Ireland, the BBC has a duty to be impartial no less than in the rest of the United Kingdom. But, as between the British army and the gunmen, the $\mathrm{BBC}$ is not and cannot be impartial ${ }^{18}$.

Pourtant, en décembre 1971, la BBC résista à la pression du gouvernement qui s'opposait à la diffusion de l'émission The Question of Ulster. Aucun membre de l'IRA ne devait y être interviewé ${ }^{19}$, mais le format consistait à reproduire une sorte de procès dans lequel plusieurs points de vue étaient exprimés sur la crise que connaissait l'Irlande du Nord. Les critiques visaient aussi le fait de divertir les téléspectateurs en mettant en scène une situation contemporaine dans laquelle des citoyens étaient tués par des bombes ${ }^{20}$.

Pendant le reste des années 1970, aucun paramilitaire républicain en activité ne fut interviewé jusqu'à ce que des journalistes de Tonight s'entretiennent, en 1979, avec des membres de l'Irish National Liberation Army. L'INLA, un groupe paramilitaire républicain créé quatre ans plus tôt par d'anciens membres de l'IRA Officielle, devint illégale au Royaume-Uni la semaine même où l'émission fut diffusée ${ }^{21}$. Quelques mois plus tôt, Airey Neave, porte-parole du Parti conservateur pour l'Irlande du Nord, avait été tué dans un attentat à la voiture piégée organisé par l'INLA.

16 Peu de temps après, une équipe de Panorama, émission phare de la BBC depuis 1953, filmait un barrage routier mis en place par l'IRA dans le village nord-irlandais de 
Carrickmore. Pour la première fois, le Prevention of Terrorism Act était invoqué et appliqué pour permettre à la police de saisir une copie du film qui n'avait pas encore été diffusé2 ${ }^{2}$. L'affaire entraîna un tollé général au Parlement ${ }^{23}$ et dans la presse britannique, Margaret Thatcher demandant à la BBC de mettre de l'ordre dans sa maison ${ }^{24}$.

Il apparaît toutefois que l'attaque lancée contre l'audiovisuel par le gouvernement conservateur n'était pas seulement inspirée par la couverture de l'Irlande du Nord. En 1982, la couverture de la Guerre des Malouines et celle de la grève des mineurs en 1984/85 suscitèrent de nombreuses polémiques ${ }^{25}$. Elle allait de pair avec une volonté de privatiser le service audiovisuel public et une accusation générale que les journalistes de la $\mathrm{BBC}$ étaient politiquement à gauche. Margaret Thatcher ne manquait d'ailleurs pas, dès que l'occasion se présentait, d'adresser différentes menaces à la Corporation en lui rappelant que la source principale de ses revenus, que constituait la redevance, n'était guère éternelle ${ }^{26}$.

En 1985, la diffusion de Real Lives: At the Edge of the Union relança les polémiques ${ }^{27}$. Cette émission dressait le portrait, au quotidien, de deux élus de (London)Derry, Gregory Campbell et Martin McGuinness, à savoir des députés du DUP et du Sinn Féin, partis politiques considérés comme les devantures des activités paramilitaires loyalistes et républicaines respectivement. Alors que l'attention publique était essentiellement focalisée sur les républicains et que le discours officiel assimilait l'IRA au Sinn Féin, il était attendu que leurs membres soient représentés de manière hostile ${ }^{28}$. Or, dans cette émission, les deux hommes évoluaient dans des contextes politiques et domestiques, et les deux exprimaient leur soutien à la violence politique. La principale polémique naquit du fait que Martin McGuinness apparaissait dans cette émission à la fois comme un homme politique légitime et comme un homme ordinaire, assis chez lui avec l'un de ses enfants sur ses genoux.

19 Avant la diffusion de l'émission, un journaliste du Sunday Times demanda à Margaret Thatcher comment elle réagirait si le « chef de l'IRA » était interviewé à la télévision. Cette même question fut posée au ministre de l'Intérieur, au secrétaire d'État pour l'Irlande du Nord ainsi qu'à deux membres du conseil d'administration de la BBC, lesquels n'avaient pas eu connaissance de cette émission. En réalité, l'émission avait déjà été visionnée par quelques dirigeants de la chaîne, mais à la suite de l'article du Sunday Times, tout le conseil de direction la regarda, à l'exception du Directeur-général Alasdair Milne, qui était en vacances. Un profond désaccord s'ensuivit entre les dirigeants et les administrateurs, lesquels voulaient interdire la diffusion de l'émission, ce qui leur valut des félicitations de la part du Premier Ministre et du ministre de l'Intérieur. Le jour où l'émission aurait dû être diffusée, les journalistes entamèrent une grève de $24 \mathrm{~h}$. Le Directeur général hésita à démissionner, ce que fit le responsable de la chaîne chargé de la couverture de l'Irlande du Nord avant de changer d'avis ${ }^{29}$.

Il existait pourtant au sein de la BBC un système de référence interne instauré en 1980. Tous les éléments relatifs à l'Irlande du Nord devaient être soumis aux supérieurs, voire aux directeurs généraux si cela était nécessaire ${ }^{30}$. Ce système était consolidé par un ensemble complet de directives. Celles de la $\mathrm{BBC}$ portaient notamment sur le langage à employer :

ARMY: We call it the army and its personnel soldiers, not the British army or British soldiers when they are in Northern Ireland. 
THE IRISH REPUBLICAN ARMY: it is never acceptable to call them that, it is acceptable to call them the Provisionals but never the Provos. Don't give pet names to terrorists... don't speak of IRA volunteers, we don't know why they joined ${ }^{31}$.

\section{Irlande du Nord furent interdites, reportées ou modifiées en raison de pressions} Irlande du émissions abordant la question du conflit en internes et externes ${ }^{38}$.

actì les membres actifs l'IRA n'étaient pas interviewés et la couverture du Sinn Féin était à la fois minime et généralement hostile ${ }^{39}, 1988$ allait marquer un nouveau tournant dans la représentation du conflit en Irlande du Nord avec 
l'instauration de la broadcasting ban, une censure télévisuelle qui conditionna la représentation du conflit pendant plusieurs années ${ }^{40}$.

\section{La Broadcasting ban}

Margaret Thatcher affirmait que, dans une guerre, les libertés devaient temporairement être mises en suspens pour vaincre l'ennemi ${ }^{41}$. Pendant six ans, les chaînes britanniques n'ont, par l'autorité de la loi, pas pu couvrir librement la situation en Irlande du Nord. Entre 1988 et 1994, elles furent privées de la liberté de faire entendre la voix des représentants des organisations interdites en Irlande du Nord et en Grande-Bretagne, ainsi que celles des représentants de trois partis politiques (Sinn Féin, Republican Sinn Féin et Ulster Defence Association). L'instauration de la broadcasting ban résulte à la fois des tensions susmentionnées entre le gouvernement et l'audiovisuel et d'une escalade de la violence paramilitaire en Irlande du Nord ${ }^{42}$.

Le 6 mars 1988, les forces spéciales britanniques, les SAS, tuaient à Gibraltar trois personnes membres de l'IRA soupçonnées de préparer un attentat. Lors de leurs funérailles au cimetière de Milltown à Belfast, un paramilitaire loyaliste attaquait le cortège, faisant trois victimes et plus de cinquante blessés. Trois jours plus tard, lors des funérailles de l'une des victimes de Milltown, une voiture s'élançait à vive allure sur le cortège. Craignant une nouvelle attaque loyaliste, les personnes présentes encerclèrent le véhicule, extirpèrent et frappèrent ses occupants. Les deux hommes furent ensuite déplacés sur un terrain vague et exécutés par des membres de l'IRA. Il s'avéra qu'il s'agissait de soldats britanniques en civil mais armés. La scène, immortalisée par plusieurs caméras, fut diffusée le soir même à la télévision. Le 22 mars 1988 , le chef de la police nord-irlandaise demandait à la BBC et à ITV de lui remettre les bandes vidéo de l'attaque qui n'avaient pas été diffusées ; ce que les chaînes refusèrent. Le lendemain, Thatcher déclarait :

I believe that everyone, the media included, has a bounden duty to do everything they can to see that those who perpetrated the terrible crimes, which we saw on television, which disgusted the whole world, are brought to justice. Either one is on the side of justice in these matters, or one is on the side of terrorism ${ }^{43}$.

Quelques semaines plus tard, Thames Television diffusait Death on the Rock ${ }^{44}$, une émission qui allait exacerber les tensions entre le gouvernement et l'audiovisuel, et plus généralement bouleverser la couverture médiatique du conflit ${ }^{45}$. Il y était affirmé que, contrairement à ce qui avait été rapporté par les premières déclarations officielles, les trois membres de l'IRA tués à Gibraltar n'étaient ni armés, ni en train de déposer une bombe dans une voiture. Toujours en 1988, en août, l'IRA faisait exploser une bombe lors du passage d'un bus de soldats britanniques sur une route de Ballygawley en Irlande du Nord, entraînant la mort de huit d'entre eux ; une autre bombe ciblait en septembre 1988 la maison du directeur de la fonction publique nord-irlandaise.

Ces événements entraînèrent l'introduction de la broadcasting ban, que le gouvernement justifia à l'aide d'un discours sécuritaire axé sur la lutte contre le terrorisme ${ }^{46}$. Le 19 octobre 1988, Douglas Hurd, ministre de l'Intérieur britannique, présenta devant la Chambre des communes les notices qu'il avait adressées aux directeurs de la BBC et à l'IBA, chargés de surveiller et d'encadrer les chaînes de télévision respectivement publiques et commerciales. Il leur était demandé : 
[...] to refrain at all times from sending any broadcast matter which consists of or includes-

any words spoken, whether in the course of an interview or discussion or otherwise, by a person who appears or is heard on the programme in which the matter is broadcast where-

(a) the person speaking the words represents or purports to represent an organization specified in paragraph 2 below [...].

31 La fonction inédite de la broadcasting ban qui posa concrètement problème aux chaînes, fut l'interdiction pesant sur les partis politiques, en particulier sur le Sinn Féin, qui comptait des membres élus au sein de la communauté locale et au sein de Parlement britannique, même si ces élus ne siégeaient pas à Westminster, dont ils ne reconnaissaient pas la légitimité. C'est sur ce point que les directives émises par les chaînes après la publication des notices portèrent spécifiquement. La BBC fit d'abord une interprétation très large de la notice, interdisant même de citer les déclarations des groupes concernés, y compris lorsque celles-ci étaient faites dans le cadre de procès. Les incertitudes de la BBC apparaissent notamment dans la formulation employée :

The government order applies also, we believe, to reports by news-readers, presenters, correspondents and reporters in the sense that they will not be able to quote what the banned organisations or other people say if those quotes support any of the organizations ${ }^{47}$.

32 À bien y regarder, les chaînes britanniques procédèrent à une application plutôt littérale des notices ministérielles, comme pour mieux les contourner. À la lecture du texte de l'interdiction, seuls les «mots prononcés» («any words spoken ») par les membres et les sympathisants des organisations proscrites ne pouvaient pas être entendus par les téléspectateurs, ce qui offrait aux journalistes l'opportunité d'emprunter une voie subsidiaire, permettant de se placer à la frontière imposée par l'interdiction sans pour autant la franchir, par le recours au sous-titrage ou au doublage par un acteur. Dans les directives de la BBC de 1989, cette approche fut explicite : « Pictures of [representatives of the proscribed organisations] speaking can be shown but the words must be spoken in voice-over by reporter or presenter or shown in caption ${ }^{48}$.

Quelques mois plus tard, la broadcasting ban fut même tournée en dérision. En novembre 1990, BBC 1 s'intéressa à la vie quotidienne des prisonniers républicains et loyalistes incarcérés à la prison de $\mathrm{Maze}^{49}$. En principe, leurs paroles n'étaient pas doublées lorsqu'ils s'exprimaient en leur nom propre, mais elles l'étaient lorsqu'ils parlaient en qualité de représentants de l'une des organisations interdites. Cette règle fut appliquée à la lettre lorsque la voix d'un prisonnier, représentant de l'IRA pour les questions alimentaires dans la prison, fut doublée alors qu'il évoquait un point aussi anodin que la taille des saucisses qui leur étaient servies.

34 Le renforcement de l'autocensure des journalistes et plus généralement des chaînes est néanmoins une conséquence de la broadcasting ban. Plusieurs projets furent abandonnés avant ou après leur réalisation. Paul Hamann, alors producteur exécutif des documentaires de la $\mathrm{BBC}$, déclarait avoir renoncé à diffuser plusieurs émissions même si elles proposaient un nouveau regard sur l'Irlande du Nord ${ }^{50} . I l$ fallut attendre 1994 pour que la broadcasting ban soit levée. Après être parvenu à amener le Sinn Féin à la table des négociations, et à obtenir un cessez-le-feu unilatéral de la part de l'IRA, le gouvernement pouvait difficilement trouver une justification aux restrictions qu'il avait imposées six ans plus tôt et qui visaient principalement les républicains. 


\section{Programmation sur BBC 1 et BBC 2 (1988-1995)} du Nord ${ }^{53} .114$ d'entre elles furent diffusées sur BBC 1 (49) et BBC 2 (65), incluant 81 documentaires (38 diffusés sur BBC1, dont 17 épisodes de Panorama). Parmi ceux-ci, 40 peuvent être considérés comme «très pertinents ». Ces documentaires portent sur trois thématiques principales qui évoluent en fonction du contexte. Plusieurs d'entre eux sont consacrés à la police en Irlande du Nord. Mais, dès la mise en place de la broadcasting ban, ces investigations évoluent pour déterminer si une politique de «shoot-to-kill», d'exécution systématique existait. Panorama consacre ainsi deux épisodes à l'éventualité d'une collusion entre les forces britanniques et les paramilitaires loyalistes. En février 1990, le premier épisode, Ulster's Regiment: A Question of Loyalty?54, soupçonna des membres de l'Ulster Defence Regiment d'avoir fourni les renseignements qui ont conduit à l'assassinat de jeunes catholiques par des paramilitaires loyalistes. Dans le second épisode diffusé en juillet 1991, Lethal Force ${ }^{55}$, John Ware, journaliste célèbre ayant une connaissance approfondie du conflit, envisagea la possibilité que les forces de sécurité en Irlande du Nord couvrent des assassinats pour masquer une politique de «shoot-to-kill». Cette série de documentaires cessa à la suite d'une polémique suscitée par la diffusion par Channel 4 de Dispatches ${ }^{56}$ sur la même thématique. La personne qui avait mené les recherches pour l'émission fut arrêtée, interrogée et accusée de parjure, avant que les charges ne soient finalement abandonnées quelques mois plus tard ${ }^{57}$.

Surtout, la question du quotidien en Irlande du Nord émerge au début des années $1990^{58}$, thématique qui avait jusque-là été peu abordée à la télévision. À l'approche des cessez-le-feu de 1994, quelques documentaires marquent le $25^{\mathrm{e}}$ anniversaire du début des «Troubles» et s'interrogent sur les chances de voir une paix durable. L'heure est à la commémoration des victimes.

Vingt-sept films et téléfilms, diffusés en particulier sur BBC 2, portent majoritairement sur l'IRA. On y assiste à des histoires d'amour impossibles entre catholiques et protestants ou des membres de l'armée ${ }^{59}$. Un thème récurrent est également celui de l'informateur au sein de l'IRA. Dans Crossfire ${ }^{60}$, un ingénieur informaticien est envoyé à Belfast pour identifier une taupe de l'IRA. Dans Final Run, un ancien membre de l'IRA devient informateur et se réfugie en Angleterre, après avoir été emprisonné pour terrorisme $^{61}$. La représentation de l'IRA est constante sur toute la période: ses membres sont inéluctablement décrits comme des terroristes, redoutables car très violents, assoiffés de vengeance, et qui, malgré leur repentir ou leur aspiration à une vie meilleure, sont condamnés à un destin tragique.

Revue Française de Civilisation Britannique, XXVI-1 | 2021 
40 D'un point de vue qualitatif, la médiatisation se focalisa sur les acteurs du conflit, en particulier sur les mouvements favorables à une réunification de l'Irlande et les autorités, au détriment des mouvements unionistes. Si la réalité du conflit et de la vie en Irlande du Nord fut abordée à l'écran jusqu'en 1994, ces thèmes s'estompèrent ensuite. Le manque d'intérêt porté au conflit même est flagrant : les chaînes semblent prêter plus d'attention au sensationnel que leur fournissent les mouvements proréunification. Il fut en effet plus aisé d'attirer les téléspectateurs en leur proposant le témoignage d'un ancien membre de l'IRA qu'en diffusant une émission sur les méandres politiques de l'exécutif nord-irlandais, ou sur le chômage en Irlande du Nord. Seulement trois émissions de la BBC, à savoir trois épisodes de Panorama, furent consacrées aux chances de parvenir à trouver un accord de paix ${ }^{62}$. La nécessité de couvrir l'actualité en Irlande du Nord tout en cherchant à faire de l'audience aurait guidé les chaînes britanniques, répondant plus à des logiques commerciales qu'auparavant.

41 Il ressort par ailleurs du répertoire d'émissions que si BBC 2 a consacré le plus d'émissions au conflit en termes de nombre, $\mathrm{BBC} 1$ a réalisé une médiatisation plus approfondie du conflit en Irlande du Nord. Cette médiatisation est sans doute plus critique sur BBC 1 que sur BBC 2, laquelle propose des émissions plus superficielles. Plus d'un tiers des émissions pouvant être qualifiées de "très pertinentes" correspondent à des épisodes que les émissions d'actualité phares des différentes chaînes de télévision ont consacrées au conflit en Irlande du Nord, notamment Panorama sur BBC 1.

Il faut en dernier lieu rappeler que le conflit en Irlande du Nord est un sujet qui attire peu les téléspectateurs de la Grande-Bretagne. En effet, ceux-ci ont montré une lassitude ${ }^{63}$ à l'égard de ce conflit auquel ils prêtaient peu d'attention, et ce dès son apparition sur les écrans. Pourtant, l'Irlande du Nord fait partie intégrante du Royaume-Uni. Nous avons constaté que la diffusion de plusieurs émissions coïncide avec des événements récents en Irlande du Nord. Cette mission d'information est la motivation la plus fréquente justifiant la diffusion des émissions répertoriées.

Les chaînes cherchèrent également à faire de l'audience et à rendre les émissions plus attractives pour y parvenir, malgré le sujet traité. Le recours aux fictions, qui attirent généralement plus de téléspectateurs que les documentaires, n'était pas suffisant : en découvrant dans le synopsis que l'émission abordait la situation en Irlande du Nord, présente ou passé, le téléspectateur se serait détourné. Les chaînes ont mis en avant les caractères inédits et exclusifs de leurs émissions, en renforçant ainsi leur aspect sensationnel. Il pouvait s'agir d'informations nouvelles ou plus souvent de personnes de renom invitées à apporter leur témoignage. Par exemple, le 24 novembre 1992, BBC 1 proposa un reportage sur un homme ayant intégré les forces spéciales de la RUC lorsqu'il avait 17 ans avant de rejoindre une unité active de l'IRA. Pour attirer les téléspectateurs, le synopsis de l'émission proposé dans The Times insista sur le caractère exclusif de ce témoignage d'un agent britannique ayant infiltré l'IRA et étant toujours en vie, en ce sens qu'il n'avait pas été tué par l'IRA en représailles à sa trahison ${ }^{64}$.

La télévision a aussi pu être utilisée pour sa capacité à toucher une grande partie de la population britannique et la sensibiliser à des questions ponctuelles, constituant ainsi un espace au sein duquel une voix alternative à celle des autorités a pu être exprimée dans le but de corriger des inexactitudes et de dénoncer des injustices. À bien y regarder, cette démarche ne fut que partiellement efficace. Sur la forme, ces émissions 
furent diffusées à des créneaux horaires privilégiés, pendant lesquels l'audience était potentiellement maximale, ce dans le but de toucher le plus grand nombre. Certaines émissions donnèrent lieu à de vives polémiques, sans permettre de réelles avancées au niveau du conflit ou de sa compréhension, et affectant même les relations entre les chaînes et l'État ${ }^{65}$. L'impact de ces émissions fut parfois plus important, allant jusqu'à conduire à la réouverture de procès et à la révision de condamnations judiciaires. Ce n'est toutefois pas sur la BBC que ces émissions furent diffusées mais sur ITV ${ }^{66}$.

\section{Conclusion}

La représentation par la BBC du conflit en Irlande du Nord entre 1969 et 1995 fut fortement alignée sur le discours officiel selon lequel le terrorisme était la cause du conflit qui opposait des républicains et des unionistes irréconciliables. Il est difficile d'affirmer que la BBC a pu librement assurer la couverture des événements en Irlande du Nord, dans la mesure où la télévision fut instrumentalisée par le gouvernement pour contrôler cette représentation. La sélection des informations, le recours à la loi ainsi que l'intimidation des journalistes sont autant de stratégies qui furent déployées par les autorités et qui eurent pour effet inéluctable de fausser la médiatisation du conflit. Cette stratégie mêlant propagande, autocensure et censure attisa les tensions entre le gouvernement et l'audiovisuel. Très tôt, on assista à une couverture partielle et partiale du conflit, laquelle conduisit à une incompréhension durable de ce dernier en GrandeBretagne.

\section{BIBLIOGRAPHIE}

BARNETT Steven, The Rise and Fall of Television Journalism: Just Wires and Lights in a Box? (Londres, Bloomsbury, 2011).

BUTLER David, The Trouble with Reporting Northern Ireland. The British State, the Broadcast Media and Nonfictional Representation of the Conflict (Aldershot, Avebury, 1995).

CATHCART Rex, The Most Contrary Region. The BBC in Northern Ireland 1924-1984, (Belfast, The Blackstaff Press Limited, 1984).

CORCoRAn Mary P. et o'BRIEN Mark (dir.), Political Censorship and the Democratic State, (Dublin, Four Courts Press, 2005).

CURTIS Liz, Ireland: The Propaganda War (Londres, Campaign for Press and Broadcasting Freedom, 1993).

KUHN Raymond, «British Television under Thatcher: A Case of Reactionary Radicalism », Revue française de civilisation britannique, 1990, vol. 5, $\mathrm{n}^{\circ}$ 4, p. 105-120.

MILLER David, Don't Mention the War: Northern Ireland, Propaganda and the Media, (Londres, Pluto Press, 1994). 
QUÉRÉ Mireille, « Les voies du silence », Revue française de civilisation britannique, 1990, vol. 5, n 4, p. 5975.

ROLSTON Bill, The Media and Northern Ireland, (Basingstoke, Macmillan, 1991).

ROLSTON Bill et MILLER David (dir.), War and Words: the Northern Ireland Media Reader, (Belfast, Beyond the Pale Publications, 1996).

SAVAGE Robert J., The BBC's 'Irish troubles' Television, Conflict and Northern Ireland, (Manchester, Manchester University Press, 2015).

VIERA John David, "Terrorism at the BBC: the IRA on British Television ", Journal of Film and Video, 1988 , vol. $40, \mathrm{n}^{\circ} 4$, p. $28-36$.

\section{NOTES}

1. WOLTON Dominique, War game. L'information et la guerre, Paris, Flammarion, 1991, p. 3.

2. V. notamment HOFFMAN Bruce, Inside Terrorim, New York, Columbia University Press, 2006, p. 176 et $\mathrm{s}$

3. Sur l'impartialité dans les médias britanniques, v. BARNETT Steven, The Rise and Fall of Television Journalism: Just Wires and Lights in a Box?, Londres, Bloomsbury, 2011, p. 227 et s. ; HARRISON Martin, TV News: Whose Bias?, Hermitage, Policy Journals, 1985 ; STARKEY Guy, Balance and Bias in Journalism: Representation, Regulation and Democracy, Basingstoke, Palgrave Macmillan, 2007.

4. PETTITT Lance, Screening Ireland: Film and Television Representation, Manchester, Manchester University Press, 2000, p. 227 ; CATHCART Rex, The Most Contrary Region. The BBC in Northern Ireland 1924-1984, Belfast, The Blackstaff Press Limited, 1984, p. ix.

5. SAVAGE Robert J., The BBC's 'Irish troubles' Television, Conflict and Northern Ireland, Manchester, Manchester University Press, 2015, p. 2.

6. Idem p. 1 et CATHCART Rex, The Most Contrary Region: The BBC in Northern Ireland 1924-1984, op. cit., p. 210.

7. CATHCART Rex, The Most Contrary Region: The BBC in Northern Ireland 1924-1984, op. cit., p. 211

8. MILLER David, Don't Mention the War: Northern Ireland, Propaganda and the Media, Londres, Pluto Press, 1994, p. 28.

9. V. CATHCART Rex, The Most Contrary Region. The BBC in Northern Ireland 1924-1984, op. cit., p. ix.

10. V. BUTLER David, The Trouble with Reporting Northern Ireland. The British State, the Broadcast Media and Nonfictional Representation of the Conflict, Aldershot, Avebury, 1995, p. 1-2 et p. 59.

11. CURTIS Liz, Ireland: The Propaganda War, Londres : Campaign For Press And Broadcasting Freedom, 1993, p. 18, et du même auteur « British Broadcasting and Ireland », Screen, 1986, n²7, p. 47.

12. MILLER David et MCLAUGHLIN Greg, "Reporting the Peace in Ireland », in ROLSTON Bill et MILLER David (dir.), War and Words: the Northern Ireland Media Reader, Belfast, Beyond the Pale Publications, 1996, p. 422-423.

13. V. o'CARROLl Lisa, «The Truth behind Real Lives", The Guardian, 12 décembre 2005 ; SEYMourURE Colin, The British Press and Broadcasting since 1945, Oxford, Blackwell Publishers, 1996, 2e éd. (1991, 1ère éd), p. 243 et s. Sur le recours à la loi pour influencer les médias, v. aussi ELDRIDGE John, KITZINGER Jenny et WILLIAMS Kevin, The Mass Media and Power in Modern Britain, Oxford, Oxford University Press, 1997, p. 12 et s.; QUÉRÉ Mireille, "Les voies du silence », Revue française de civilisation britannique, 1990, vol. 5, $\mathrm{n}^{\circ}$ 4, p. 62 et s.; SERGEANT Jean-Claude, La Grande-Bretagne de Margaret Thatcher, Paris, Presses Universitaires de France, 1994, p. 164-167. 
14. KUHN Raymond, "British Television under Thatcher: A Case of Reactionary Radicalism ", Revue française de civilisation britannique, 1990, vol. 5, $\mathrm{n}^{\circ}$ 4, p. 105-120.

15. Un récit plus détaillé des événements figure dans le chapitre 8 de Philip SCHLESINGER, Putting 'Reality' Together: BBC News, Londres, Methuen, 1987, $2^{\text {nde }}$ édition ( 1 ère ed. en 1978)

16. BRAUN Edward, "What Truth is There in this Story? ", in BIGNELL Jonathan et LACEY Stephen (dir.), British Television Drama: Past, Present and Future, Basingstoke, Palgrave Macmillan, 2014, p. 172-173.

17. CATHCART Rex, The Most Contrary Region. The BBC in Northern Ireland 1924-1984, op. cit., p. 229.

18. HILL Charles, Behind the Screen: The Broadcasting Memoirs of Lord Hill, Londres, Sidwick \& Jackson, 1974, p. 219-221.

19. MILLER David, Don't Mention the War: Northern Ireland, Propaganda and the Media, op. cit., p. 29.

20. BRIGGS Asa, The History of Broadcasting in the United Kingdom, vol. V: Competition, Oxford, Oxford University Press, 1995, p. 909.

21. MILLER David, Don't Mention the War: Northern Ireland, Propaganda and the Media, op. cit., p. 34.

22. Pour un récit plus détaillé, voir CURTIS Liz, Ireland: The Propaganda War, London : Pluto Press, 1984, p. 164-182.

23. Voir les débats très instructifs du $1^{\mathrm{er}}$ août 1980 interrogeant le positionnement de la $\mathrm{BBC}$ : "There is much public concern about the behaviour of the agents of the media in relation to criminals. (...) Terrorism thrives on publicity. (...) Last year two incidents occurred in which the BBC, acting through its staff, in my opinion, broke the law. (...) The conduct of the BBC staff involved in this matter was disgraceful and, I believe, criminal. What the $B B C$ and its apologists fail to remember is that in Northern Ireland we are fighting a vicious war in which British lives are being lost almost every day. It is a war with battles just as bitter and cruel as any during the Second World War » Hansard, $1^{\mathrm{er}}$ août 1980, col. 2002 et 2003.

24. "I think that it is time that the BBC put its house in order", Hansard, 8 novembre 1979, col. 606-613.

25. Pour un récit détaillé des polémiques suscitées par leur couverture médiatique, v. PHILo Greg, Seeing and Believing. The Influence of Television, Londres, Routledge, 1990 ; QUÉRÉ Mireille, « Les voies du silence », op. cit., p. 71 ; GLASGOW UNIVERSITY MEDIA GROUP, War \& Peace News, op. cit.; PARSONS Michael, «Les Malouines : une guerre de propagande ?», in DICKASON Renée et CERVANTES Xavier, La propagande au Royaume-Uni, De la Renaissance à l'Internet, Paris, Ellipses, 2002.

26. V. ELDRIDGE John, KITZINGER Jenny et WILLIAMS Kevin, The Mass Media and Power in Modern Britain, op. cit., p. 54-55.

27. Real Lives: At the Edge of the Union, $\mathrm{BBC} 1,16$ octobre 1985. V. SEYMOUR-URE Colin, The British Press and Broadcasting since 1945, op. cit., p. 236 ; LEAPMAN Michael, "The 'Real Lives' Controversy ", in ROLSTON Bill et MILLER David (dir.), War and Words: the Northern Ireland Media Reader, préc., p. 96-117 ; QUÉRÉ Mireille, « Les voies du silence », op. cit., p. 72 ; BARNETT Steven, The Rise and Fall of Television Journalism: Just Wires and Lights in a Box?, op. cit., p. 91 et s. V. aussi VIERA John David, « Terrorism at the BBC: the IRA on British Television ", Journal of Film and Video, 1988, vol. 40, n 4, p. 28-36.

28. V. CATHCART Rex, The Most Contrary Region. The BBC in Northern Ireland 1924-1984, op. cit., p. 229.

29. MILNE Alasdair, DG: The Memoirs of a British Broadcaster, Londres : Coronet, 1989, p. 188 et 190

30. KELLY Mary et ROLSTON Bill, «Broadcasting in Ireland: Issues of National Identity and Censorship ", in CLANCY Patrick, DRUDY Sheelagh, LYNCH Kathleen et o'DOWD Liam (dir.), Irish Society: Sociological Perspectives, Dublin, Institute of Public Administration, 1995, p. 584.

31. BBC Style Guide, 1989. Archives de la BBC, Caversham.

32. SEYMOUR-URE Colin, The British Press and Broadcasting since 1945, préc., p. 62.

33. Word in Action: the Taming of the Beeb, 29 février 1988, ITV.

34. Pendant l'été 1985, le gouvernement en était à un stade crucial des négociations angloirlandaises et la manière dont la télévision américaine avait traité le détournement d'un avion de la TWA par un groupe islamiste faisait polémique. On accusait les médias télévisés de favoriser les 
pirates de l'air en les interviewant et en diffusant leurs requêtes à la télévision. En faisant référence à ce détournement, $M$. Thatcher avait avancé, lors d'un discours prononcé aux ÉtatsUnis, que les médias fournissaient aux «terroristes » l'« oxygène de la publicité ». V aussi KITTHEWITT David (dir.), Crime and the Media: The Post-Modern Spectacle, Londres, Pluto Press, 1995.

35. V. FITZWALTER Raymond, The Dream That Died: The Rise and Fall of ITV, op. cit., p. 79-80.

36. Northern Ireland Criminal Law Act de 1967, le British Contempt of Court Act de 1981,le Police and Criminal Evidence Act de 1984, un nouvel Official Secrets Act voté en 1989, puis la révision, toujours en 1989, du Prevention of Terrorism Act de 1984.

37. L'existence de cette commission, fondée en 1912 dans la foulée de la loi sur les secrets officiels de 1911, ne fut révélée au public qu'en 1952. GENSANE Bernard, Censure et libertés au Royaume-Uni, Paris, Ellipses, 2001, p. 116.

38. CURTIS Liz, "A Catalogue of Censorship, 1959-1993 », op. cit., p. 42-91. V. aussi The British Media and Ireland. Truth: the First Casualty, Londres : Campaign For Press And Broadcasting Freedom, 1978.

39. MILLER David, Don't Mention the War: Northern Ireland, Propaganda and the Media, op. cit., p. 66 et p. 84 .

40. CORCORAN Mary P. et O'BRIEN Mark, «Introduction », in CORCORAN Mary P. et o'BRIEN Mark (dir.), Political Censorship and the Democratic State, op. cit., p. 18. PHILo Greg, «Television, Politics and the Rise of the New Right », in PHILO Greg (dir.), Glasgow Media Group Reader : Industry, Economy, War and Politics, vol. 2, op. cit., p. 206.

41. "To beat off your enemy in a war you have to suspend some of your civil liberties for a time " cité par OAKLEY Robin, "Now it's up to the people; Prime Minister talks about her chief concerns ", The Times, 26 octobre 1988. V. aussi SERGEANT Jean-Claude, La Grande-Bretagne de Margaret Thatcher, op. cit., p. 162-163.

42. QUÉRÉ Mireille, «Les voies du silence », op. cit., p. 71-75. L'auteur propose une chronologie de « Dix ans de rapports conflictuels entre le gouvernement, le Parti conservateur et les media ».

43. Hansard, 22 mars 1988, col. 194

44. Jeudi 28 avril 1988 sur ITV, de $21 \mathrm{~h}$ à $22 \mathrm{~h}$.

45. KUHN Raymond, «British Television under Thatcher: A Case of Reactionary Radicalism », op. cit., p. 117 et s.; BARNETT Steven, The Rise and Fall of Television Journalism: Just Wires and Lights in a Box?, op. cit., p. 91 et s.

46. THATCHER Margaret, 10 Downing Street. Mémoires, Paris : Albin Michel, 1993, p. 340 et s.

47. BBC Directive, 18 novembre 1988.

48. BBC, Guidelines for Factual Programmes, 1989, section 40.

49. Inside Story: The Maze - Enemies Within, BBC 1, mardi 27 novembre 1990, 22h20-23h20.

50. The Guardian, 15 mai 1989.

51. V. «Sources of Information on Politics and the Political Process ", Ipsos Mori, https:// www.ipsos-mori.com/researchpublications/researcharchive/1238/Sources-of-Information-onPolitics-and-the-Political-Process.aspx (consulté le 31 juillet 2020).

52. Données disponibles à partir de 1975. V. http://www.nationmaster.com/country-info/ profiles/United-Kingdom/Media (consulté le 31 juillet 2020).

53. Notre analyse d'appuie sur le répertoire des émissions sur le conflit en Irlande du Nord (émissions en direct, journaux télévisés et publicités exclus) constitué dans le cadre de travaux antérieur. V. Elodie Gallet, Médiatisation et médiation: le cas de la représentation du conflit en Irlande du nord à la télévision nationale britannique (1988-2005), Thèse Poitiers 2016, dir. Pr S. Finding, annexe $\mathrm{CD}$ « répertoire des émissions ».

54. Panorama: Ulster's Regiment a Question of Loyalty?, BBC 1, 19 février 1990, 21h30-22h20. Cet episode est disponible en ligne à l'adresse https://www.youtube.com/watch?v=9slL-rfUJDM (consulté le 30 août 2020). 
55. Panorama: Lethal Force, BBC 1, lundi 22 juillet 1991, 21h30-22h10. Cet episode est disponible en ligne à l'adresse https://www.youtube.com/watch?v=dSPUKyFL7Rg (consulté le 30 août 2020).

56. Dispatches: The Committee, Channel 4, 2 octobre 1991, 21h-22h.

57. V. CURTIS Liz, «A Catalogue of Censorship, 1959-1993 », op. cit., p. 84.

58. Heart of the Matter: School's Out, BBC 1, dimanche 7 juillet 1991, 10h40-11h15; This is the Day, BBC 1, dimanche 13 janvier 1991, 9h15-10h ; The Lowdown, BBC 1, mardi 6 avril 1993, 17h10-17h35

59. Ryan's Daughter, BBC1, vendredi 22 avril 1988, 22h20-1h30 ; Foreign Bodies, 3 épisodes diffusés en 1989 sur BBC2, jeudi 25 mai 1989, 21h-21h30, jeudi $1^{\text {er }}$ juin et jeudi 8 juin, 21h-21h30

60. $\mathrm{BBC} 121 \mathrm{~h} 30-22 \mathrm{~h} 20$ (premier épisode) puis 21h20-22h20 (épisodes 2 à 4), diffusés les mardis 15 , 22 et 29 mars, puis les 5 et 12 avril.

61. 4 épisodes diffusés en 1988 sur BBC 2 les dimanches 10 juillet (21h50-22h40), 17 juillet (22h-22h50), 24 juillet (22h-22h50), 31 juillet (22h05-22h55)

62. Lundi 29 novembre 1993, BBC1,21h30-22h10, Panorama: Talking to Mr Adams; lundi 25 juillet 1994, BBC 1, 21h30-22h10, Panorama; lundi 17 octobre 1994, BBC 1, 21h30-22h10, Panorama: the Uneasy Peace.

63. À titre illustratif, dans une critique publiée dans The Times au sujet du conflit en Irlande du Nord, on pouvait lire « «Faced with yet another series of documentaries on the conflict in Northern Ireland, the prospective viewer may well be tempted to stifle a groan and look to the other channels ", v. WAYMARK Peter, « Aspiring to Maim and Kill », The Times, 7 août 1989, p. 19.

64. Inside Story: The Informer, BBC 1, mardi 24 novembre 1992, 22h25-23h15.

65. FITZWALTER Raymond, The Dream that Died. The Rise and Fall of ITV, op. cit., p. 28 et s.

66. Le cas des Six de Birmingham est intéressant à cet égard, puisque plusieurs émissions ont dénoncé l'erreur judiciaire qui avait conduit à leur condamnation après un attentat à la bombe à Birmingham en 1974. Il fallut un épisode de "World in Action" en 1985 et une fiction documentaire pour attirer l'attention de l'opinion publique sur cette question et ouvrir un procès en appel, conduisant à la libération des six hommes. World in Action: In the Interest of Justice, ITV, 28 octobre 1985 ; Who Bombed Birmingham?, ITV, mercredi 28 mars 1990, 20h-22h.

\section{RÉSUMÉS}

La BBC a joué un rôle actif dans les conflits, à commencer par la Deuxième Guerre mondiale jusqu'à la Guerre en Irak, en passant par la Guerre des Malouines. La couverture du conflit en Irlande du Nord a toutefois suscité une difficulté inédite : ce conflit, contrairement aux autres guerres dans lesquelles les ennemis étaient étrangers, s'est déroulé sur le territoire national et impliquait non seulement l'armée mais aussi des civils britanniques.

Cet article dresse un panorama de la couverture réalisée par la BBC depuis la recrudescence de la violence à la fin des années 1960 jusqu'en 1995, en mettant l'accent sur les stratégies utilisées par le gouvernement britannique pour influencer cette couverture, frappée de 1988 à 1994 par une censure directe. La réflexion est complétée par un examen des documentaires et téléfilms diffusés sur BBC 1 et BBC 2 entre 1988 et 1995. À quelques exceptions près, la BBC a globalement adhéré à la politique gouvernementale en Irlande du Nord en proposant une couverture partielle et partiale du conflit.

The $\mathrm{BBC}$ played an active role in military conflicts, from the Second World War to the Falklands War and the war in Iraq. Nevertheless, coverage of the conflict in Northern Ireland poses 
particular difficulties, in that the conflict played out on British territory and was fought not just by the army but also by civilians.

This article sketches out a panorama of BBC coverage of Northern Ireland, from the resurgence of violent conflict in the late 1960, through to 1995 . With a few exceptions, the BBC followed government policy on the question by putting forward a partial and biassed view of the conflict.

INDEX

Mots-clés : $\mathrm{BBC}$, Irlande du Nord, censure, impartialité

Keywords : BBC, Northern Ireland, censorship, bias

AUTEUR

ELODIE GALLET

Université d'Orléans 